\title{
ON THE STRUCTURE OF SPLIT INVOLUTIVE REGULAR HOM-LIE ALGEBRAS
}

\author{
JiAN ZHANG, CHIPING ZHANG AND YAN CAO
}

\begin{abstract}
We study the strucure of arbitrary split involutive regular Hom-Lie algebras. By developing techniques of connections of roots for this kind of algebras, we show that such an algebra $L$ is of the form $L=U+\sum_{[j] \in \Lambda / \sim} I_{[j]}$ with $U$ a subspace of the involutive abelian subalgebra $H$ and any $I_{[j]}$, a well described involutive ideal of $L$, satisfying $\left[I_{[j]}, I_{[k]}\right]=0$ if $[j] \neq[k]$. Under certain conditions, in the case of $L$ being of maximal length, the simplicity of the algebra is characterized and it is shown that $L$ is the direct sum of the family of its minimal involutive ideals, each one being a simple split involutive regular Hom-Lie algebra.
\end{abstract}

Mathematics subject classification (2010): 17B05, 17B20, 17B22, 17B65.

Keywords and phrases: Involutive, Hom-Lie algebra, root system, root space.

\section{REFERENCES}

[1] J. Hartwig, D. LaRsSOn AND S. Silvestrov, Deformations of Lie algebras using $\sigma$-derivations, J. Algebra 295 (2) (2006), 314-361.

[2] F. Ammar, Z. EJBehi AND A. Makhlouf, Representations and cohomology of n-ary multiplicative Hom-Nambu-Lie algebras, J. Geom. Phys. 61 (10) (2011), 1898-1913.

[3] F. Ammar, S. Mabrouk And A. Makhlouf, Cohomology and deformations of Hom-algebras, J. Lie Theory 21 (4) (2011), 813-836.

[4] M. Elhamdadi, A. Makhlouf, Deformations of Hom-alternative and Hom-Malcev algebras, Algebras Groups Geom. 28 (2) (2011), 117-145.

[5] B. Sun, L. Chen, Rota-Baxter multiplicative 3-ary Hom-Nambu-Lie algebras, J. Geom. Phys. 98 (12) (2015), 400-413.

[6] A. Makhlouf, S. Silvestrov, Notes on 1-parameter formal deformations of Hom-associative and Hom-Lie algebras, Forum Math. 22 (4) (2010), 715-739.

[7] Y. S. Cheng, Y. C. Su, (Co)Homology and universal central extension of Hom-Leibniz algebras, Acta Math. Sin. (Engl. Ser.) 27 (5) (2011), 813-830.

[8] A. J. CALDERón, J. M. SÁnCheZ, On the structure of split involutive Lie algebras, Rocky Mountain J. Math. 44 (5) (2014), 1445-1455.

[9] A. J. CALDERón, On split Lie algebras with symmetric root systems, Proc. Indian Acad. Sci. (Math. Sci.) 118 (3) (2008), 351-356.

[10] M. J. Aragón, A. J. CAlderón, Split regular Hom-Lie algebras, J. Lie Theory 25 (3) (2015), $875-888$.

[11] A. J. CAlderón, J. M. SÁnchez, On split Leibniz algebras, Linear Algebra Appl. 436 (6) (2012), $1648-1660$.

[12] A. J. Calderón, On split Lie triple systems, Proc. Indian Acad. Sci. (Math. Sci.) 119 (2) (2009), $165-177$.

[13] Y. CaO, L. Y. Chen, On the structure of split Leibniz triple systems, Acta Math. Sin. (Engl. Ser.) 31 (10) (2015), 1629-1644. 